\title{
Del Hospício de Pedro II al Hospital Nacional de Alienados: cien años de historias (1841-1944)
}

\author{
From the Hospicio de Pedro II to the Hospital Nacional de \\ Alienados: a hundred years of records (1841-1944)
}

\author{
Allister Andrew \\ Teixeira Dias ${ }^{i}$ \\ 'Bolsista recém-doutor, Casa de \\ Oswaldo Cruz/Fiocruz. \\ Rio de Janeiro - RJ - Brasil \\ orcid.org/0000-0003-1882-4211 \\ allisterdias@hotmail.com
}

\section{Raisa Monteiro Capela ${ }^{i i}$ \\ ii Mestranda, Programa de Pós- graduação em História das Ciências e da Saúde/Fiocruz. Rio de Janeiro - RJ - Brasil orcid.org/0000-0001-5147-4597 raisacapelam@gmail.com}

Recebido em 27 mar. 2017. Aprovado em 2 jul. 2018.
DIAS, Allister Andrew Teixeira; CAPELA, Raisa Monteiro. Del Hospício de Pedro II al Hospital Nacional de Alienados: cien años de historias (18411944). História, Ciências, Saúde - Manguinhos, Rio de Janeiro, v.26, n.4, out.-dez. 2019, p.1203-1210.

\section{Resumen}

Esta nota de investigación expone perspectivas metodológicas, fuentes documentales, inscripción historiográfica y reflexiones que surgen de la investigación en curso, titulada "Del Hospício de Pedro II al Hospital Nacional de Alienados: cien años de historias (1841-1944)". Un grupo de investigadores y estudiantes asociados al proyecto han analizado la historia de la primera institución psiquiátrica de Brasil en el periodo comprendido entre la segunda mitad del siglo XIX y mediados del siglo XX. También están comprometidos con el ideal de contribuir a la organización y conservación de los fondos documentales de esta institución. Aquí presentamos el carácter innovador del proyecto, especialmente por sus perspectivas metodológicas en combinación con enfoques de preservación de documentos históricos.

Palabras clave: Hospital Nacional de Alienados; historia de Brasil; preservación documental; psiquiatría; locura.

\section{Abstract}

This research note lays out methodological approaches, documentary sources, historiographical inscription and reflections that arose from an ongoing research study entitled "From the Hospício de Pedro II to the Hospital Nacional de Alienados: a hundred years of records (1841-1944)." A group of researchers and students involved in the project have analyzed the history of the first psychiatric institution in Brazil for the period from the second half of the nineteenth century through the mid-twentieth century. They are also committed to the ideal of contributing to the organization and conservation of the documentary sources of this institution. Here we present the innovative nature of the project, especially due to its methodological approaches in combination with its focus on preserving historical documents.

Keywords: Hospital Nacional de Alienados; Brazilian history; document preservation; psychiatry; madness. 
ste proyecto de investigación tiene dos objetivos generales concatenados entre sí. El
primero de ellos es analizar el modo en que el Hospicio de Pedro II, rebautizado como Hospital Nacional de Alienados (HNA), ${ }^{1}$ primera institución de reclusión en el Brasil, especialmente orientada a los alienados, se constituyó en un centro nacional de producción y difusión de conocimientos científicos y de prácticas asistenciales relativas a la medicina mental en el pais. Durante un siglo, la institución ha servido como espacio de diálogo, disputas, negociación y experiencia para distintos sujetos sociales. Estas vivencias humanas en el interior de la institución y relativas al imaginario social de la "locura" también interesan a este proyecto.

El segundo objetivo general es incentivar acciones de preservación y divulgación de los fondos documentales del antiguo hospicio, los cuales se encuentran bajo el control de cuatro de sus instituciones herederas: el Instituto de Psiquiatría de la Universidad Federal de Rio de Janeiro (Ipub/UFRJ), el Instituto Municipal de Asistencia a la Salud Nise da Silveira (IMASNS/ SMS-RJ), el Instituto Municipal de Asistencia a la Salud Juliano Moreira (IMASJM/SMS-RJ) y el Centro de Estudios e Investigaciones de la Escuela de Gestión Penitenciaria (Secretaria de Estado de Administración Penitenciaria de Rio de Janeiro, Seap-RJ). ${ }^{2}$

El borde temporal escogido tiene como marco inicial el decreto de creación del hospicio, 18 de julio de 1841 (Brasil, 18 jul. 1841), como primera institución imperial enfocada a la asistencia de los alienados (Engel, 2001). El fin del periodo investigado se sitúa en el año 1944, cuando el decreto-ley n.7.055, de 18 de noviembre, crea el Centro Psiquiátrico Nacional (CPN), lo que supone la definitiva extinción del antiguo hospicio (Brasil, 18 nov. 1944).

Desde los estudios clásicos de Foucault, Szasz, Goffman e Laing, en el contexto internacional, y Machado y colaboradores (1978), Costa, 2007 (publicado originalmente en 1976), Cunha (1986) y Engel (2001), en el contexto brasileño, la creación de los asilos de alienados se ha interpretado en sentido contrario al conferido por la retórica oficial: la terapéutica moral - inscrita en la propia arquitectura de reclusión - no sería la expresión de un tratamiento humanitario y racional, ya que procedería del intento de dominar las conductas desviadas y reforzar el conformismo con el orden burgués y sus valores emergentes. Nuestro análisis persigue, como se verá más adelante, actualizar la historiografía sobre el asunto, teniendo como una hipótesis de investigación la centralidad de esta institución para la constitución de la psiquiatría y ciencias afines en el país (medicina legal, criminología, neurología y psicología experimental). Más recientemente, la historiografía ha matizado el énfasis otorgado a la función de control social de la psiquiatría y del hospicio (Scull, 2006).

No se trata aquí de rechazar la clasificación diagnóstica como acto político, exento de valor, sino de comprender la psiquiatría como una práctica sociocognitiva (Latour, 1995; Huertas, 2009) y relativizar la clave de análisis que la reduce a la normalización impuesta por el sistema social, a la vista de la racionalización del control sobre los sujetos desviados. Es preciso estar atento a procesos interrelacionados que corresponden a esferas distintas de la dinámica societaria en curso, cambios y contingencias se han apuntado por las narrativas atentas de instituciones donde los diferentes intereses se sobreponen e interactúan en un desarrollo continuo (Porter, Whright, 2003; Ablard, 2008). Por ello, podemos aceptar la caracterización de esta institución en una clave conceptual específica. Se trata de la idea de "institución camaleónica", al darse en ella el cruce de discursos, muy a menudo en 
oposición; o sea, con rasgos mezclados de custodia, represión, violencia, control, asistencia y preocupaciones terapéuticas e humanitarias (Sacristán, sept.-dic. 2001; Zulawsky, 2004).

La equiparación de categorías psiquiátricas con el estigma y la desviación en las conductas es comprensible y, quizás hasta cierto punto, lógica. Sin embargo, entenderlas de esa forma reduce las clasificaciones psiquiátricas a apéndices de los factores sociales, culturales e intelectuales. Es cierto que los sistemas de clasificación no son intrínsecamente evidentes por sí mismos, como tampoco los datos. Al contrario, emergen del crisol de la experiencia humana; el cambio y la variabilidad, no la inmutabilidad, son sus características (Berrios, 2012).

\section{Fuentes}

Las fuentes primarias que utilizamos son los fondos médicos (la documentación clínica) y administrativos existentes en las cuatro instituciones herederas del antiguo hospicio, referentes a su funcionamiento como lugar de ciencia y de asistencia médica en sus diferentes secciones. Este conjunto está compuesto, cuantitativamente, por la siguiente documentación:

- ocho libros de registro de entrada, salida y diagnóstico de los pacientes en el Pabellón de Observación Clínica del HNA: cuatro para hombres y cuatro para mujeres (18941944), (fondo Ipub, con el sello del Comité Nacional del Programa Memoria del Mundo, de Unesco). Del mismo fondo, 627 libros de observaciones clínicas (1896-1944) con aproximadamente 63.500 registros manuscritos sobre pacientes internados en el Pabellón de Observación (fondo Ipub);

- 168 recortes de periódicos (1909 a 1950) que abordan asuntos sobre los pacientes y en los que se describe el motivo de la internación, así como particularidades de cada caso específico (fondo Ipub);

- 28.781 informes de internación, constituidos por fichas de registro y anexos que remiten a la trayectoria de los individuos internados en el HNA (fondo IMASNS/SMS);

- 12 libros de administración del HNA, entre 1853 y 1942, (fondo IMASNS/SMS), contiendo libro de recetas; registros de gastos; registros de los conceptos de los exámenes de sanidad de los alienados en el Hospicio de Pedro II; cuenta corriente de los pacientes pensionistas; registros de los esclavos; cuadrantes de horarios de los facultativos clínicos y adjuntos del Hospicio de Pedro II; libro de registro de enfermeros/nombramientos de ayudantes de enfermeros; movimientos de los depósitos de cadáveres; movimiento del Gabinete Dental; registro de entradas y salidas de pacientes de las secciones masculinas.

- siete libros de observaciones clínicas, entre 1909 y 1944 (fondo IMASNS/SMS);

- 117 fichas de observaciones del Pabellón Bourneville (pabellón infantil): ala de los niños (1925 a 1941; fondo IMASJM/SMS);

- 91 fichas de observaciones del Pabellón Bourneville (pabellón infantil): ala de las niñas (1926 a 1941; fondo IMASJM/SMS);

- 656 fichas de observaciones de la Sección Esquirol (ala femenina; 1938 a 1942; fondo IMASJM/SMS);

- 771 fichas de observaciones de la Sección Pinel (ala masculina; 1938 a 1944, fondo IMASJM/SMS). 
En cuanto a los documentos clínicos de la Sección Lombroso del HNA y los laudos y documentos periciales, ambas colecciones se hallan custodiadas por el Museo Penitenciario de la Seap/RJ. Con el apoyo de la institución, ${ }^{3}$ el equipo se ha puesto como meta establecer negociaciones para digitalizar el material como una actividad dentro del convenio ya existente entre la Fiocruz y el Centro de Microfilmación y Digitalización (CMD) del Ministerio de Salud. Además, será necesario valorar el contenido de los informes y laudos. Estos están recogidos en libros de tapa dura, encuadernados, e incluyen la evaluación psiquiátrica de diversos pacientes del HNA y del Manicomio Judiciario del Rio de Janeiro. Para el análisis de los referidos libros, aún precisaremos identificarlos y separar el material de interés para el objeto de este estudio.

Pieza clave de la modernidad psiquiátrica brasileña, el HNA nos ha legado un gran conjunto de testimonios y fragmentos de su existencia histórica. Estos vestigios requieren de una sistemática "inmersión" para la elaboración de sentidos e interpretaciones acerca de su existencia (Schorske, 2000). Exactamente lo que propone este amplio proyecto de investigación, que busca narrar la historia de la institución por medio de sus expedientes clínicos, "fuentes históricas polifónicas" (Molina, 2009), y debido a no ceñirse solamente a las "historias clínicas", sino que también se recogen las experiencias de sus protagonistas, en especial (si bien no en exclusiva) de psiquiatras y pacientes, abre significativas posibilidades interpretativas.

\section{Objetivos y investigaciones en andamiento}

En el conjunto documental mencionado, se han investigado algunos puntos particulares. Siguen algunos ejemplos:

(a) el modo en que la medicina mental conformó parámetros epistemológicos para las terapéuticas y los diagnósticos aplicados en el HNA;

(b) el cotidiano del hospicio con objetivo de describir el recorrido de los pacientes en su interior;

(c) la manera en que la institución estuvo marcada por las jerarquías sociales prevalentes, reformulando, perpetuando y negociando los papeles sociales desempeñados por los diversos sujetos presentes en aquel microcosmos;

(d) las diferentes representaciones del HNA y la locura, tal como aparecieron en la literatura especializada, en la gran prensa, entre la población lega y los internos;

(e) la elaboración histórica de la relación entre crimen y anormalidad/patología en el HNA, con el objeto de contrastar la recepción de teorías médico-criminológicas en la construcción diagnóstica generada en la institución;

(f) el tratamiento destinado a los niños y adolescentes en el seno de la institución de reclusión;

(g) la historia de los suicidios y de las prácticas suicidas cometidas por los internos del HNA, y otras más.

Nuestras investigaciones comprenden las etapas de recopilación, selección, lectura y esquematización de la documentación relativas a la investigación propiamente dicha, a saber: el análisis y catalogación de los instrumentos de investigación ya existentes; la selección de documentos clínicos, administrativos, bibliográficos e iconográficos del HNA 
y de sus personajes; la selección, lectura y esquematización de los textos teóricos, históricos e historiográficos; la lectura y esquematización de las fuentes primarias; el análisis de los datos recogidos. También, la configuración y el entrenamiento para el uso de herramienta tecnológica, elaborada a partir del sistema AtoM, con la inserción de los datos archivísticos en esta herramienta tecnológica.

Se pueden enumerar algunos resultados técnicos. Se ha efectuado la higienización, identificación y organización de la documentación del HPII y del HNA existente en el IMASJM, con actuación de los archivistas que trabajan en la institución (Pereira et al., 2016). ${ }^{4}$ En curso está la descripción y alimentación de la base con esta documentación, así como iniciativas para la preservación de la misma. Se recogieron 439 encuardenaciones, siendo cincuenta del siglo XIX, 17 sin identificación de fecha y 372 del siglo XIX (69 fuera del alcance temporal de la investigación). Para la investigación, destacamos la identificación de "Libros de empleados", "Libros de ocurrencias", "Libros de enfermedades somáticas y tratamientos" y "Libros de observación de hombres y mujeres". Todos ellos, desde mediados del siglo XIX hasta la década de 1940, relativos a varias secciones de la institución.

En el Museo Penitenciario, vinculado a la Seap/RJ y que posee la custodia de la documentación relativa al "Servicio de Locos Delinquentes", identificamos cinco series documentales, con documentación administrativa, clínica y bibliográfica. Se ha hecho la higienización, identificación y descripción de la documentación clínica (libros de observación de pacientes del referido "Servicio") y, en el momento, está en marcha el mismo proceso para la documentación administrativa.

\section{Los procedimientos técnicos para organización y preservación documental}

Uno de los ejes de este proyecto es el de servir como catalizador para la puesta en marcha de una herramienta tecnológica que permita optimizar la recuperación de las informaciones a las que acceden los investigadores, interesados en el contenido de este material, a través de la adopción de procedimientos de descripción archivística, según las normas nacionales e internacionales. Tal herramienta será compartida, tanto en lo tocante a la tecnología como a la información, por todas las instituciones implicadas. Para elaborarla, estamos utilizando el sistema AtoM, un software libre, cuya configuración ya ha sido ejecutada por técnicos de Casa de Oswaldo Cruz, teniendo en consideración la especificidad de las fuentes documentales del área de la salud.

Esto supone, en consecuencia, la posibilidad de sistematizar informaciones sobre el fondo, con la creación de mecanismos de control que faciliten su accesibilidad. Un sistema gratuito, desarrollado con herramientas de código abierto (Apache, MySQL, PHP e Symfony) que permite la generación de un ambiente "multinivel" en conformidad con la Norma Brasileña de Descripción (Nobrade), lo que a su vez posibilita un acceso multilingüe y la unión de fondos de diferentes instituciones archivísticas. De este modo, la elección viene determinada tanto por los reducidos costes de instalación, configuración y mantenimiento, como por la perspectiva de dialogar con otras instituciones que utilizan el mismo sistema o vayan a utilizarlo. Y admite, asimismo, el desdoblamiento de acciones de accesibilidad, con vistas a la posterior digitalización del fondo, en todo o en parte. 


\section{Consideraciones finales}

Nuestro proyecto pretende avanzar sobre todo por la investigación de la relación entre teoría y práctica psiquiátrica, percibiendo las recepciones y usos de categorías diagnósticas y teorías en circulación en la praxis clínica, revelada a su vez en los libros de observación de pacientes. De ahí la relevancia de un enfoque que cruza lo cuantitativo y lo cualitativo en el estudio de las instituciones, ampliamente basadas en la exploración de los expedientes clínicos de las instituciones psiquiátricas (Molina, 2009; Huertas, 2012; Dörries; Beddies, 2003). Traemos una mirada más de cerca a la praxis clínica, cómo acciona ciertos patrones clasificatorios, y cómo amalgama el corpus teórico con concepciones de la moralidad vigente (Berrios, 2012). Además, una atención especial se ha orientado hacia la manera en que la práctica psiquiátrica opera con y ayuda a construir un lenguaje "generificada", dando definiciones normativas de feminidad y masculinidad (Tomes, 1994; Engel, 2008).

Algunos trabajos recientes han mirado hacia el HNA de maneras más matizadoras y complejas, mostrando cómo esta institución fue, a lo largo de su historia, atravesada por variadas intenciones y concepciones acerca de las perspectivas de asistencia, producción de conocimiento y control y gestión social (Gonçalves, 2013; Ribeiro, 2016; Dias, 2011). También hemos profundizado este punto, sobre todo en lo que se refiere al cotidiano de la institución y a las demandas sociales en la definición de las internaciones psiquiátricas. El conocimiento sobre el cotidiano institucional será incrementado, valiéndose de la polifonía de las fuentes clínicas y de sus anexos: cartas y escritos diversos de pacientes y parientes, recortes de periódicos, entre otros. La investigación ha avanzado en el entendimiento de las representaciones sociales más amplias acerca del HNA y de la psiquiatría. Además, el foco ha recaído, hasta el momento, en el estudio de la pluralidad de terapias implementadas en la institución y las transformaciones por las cuales las mismas pasaron, sobre todo, en el Pabellón de Observaciones.

En resumen, proponemos la investigación histórica y la conservación de los fondos archivísticos de las instituciones psiquiátricas como indisociables. Una investigación con tales objetivos debe necesariamente desdoblarse en varias direcciones. En primer lugar, se trata de cuestionar la psiquiatría en su contexto, explorando las relaciones entre la cultura y algunos criterios de la historia de las ciencias, como la recepción, la circulación, la apropiación, etc. En segundo lugar, es pertinente investigar las prácticas, actores, lugares y momentos en torno a los cuales pudo establecerse la psiquiatría nacional. En tercer lugar, la investigación propicia la aglutinación de las fuentes y objetivos de cada investigador, a fin de ser útil para todos los interesados en esta área de estudios y, dentro de este proceso, pretende proteger ese acervo riquísimo en aras de salvaguardarlo para permitir la multiplicación de investigaciones.

\section{AGRADECIMIENTOS}

Este artículo es el resultado de la investigación desarrollada en el marco del proyecto "Do Hospício de Pedro II ao Hospital Nacional de Alienados: cem anos de história (1841-1944), que contó con financiación de Proep-CNPq/Fiocruz n.440832/2015. 


\section{NOTAS}

${ }^{1}$ La institución fue inaugurada como Hospício de Pedro II durante el Imperio (1852-1889) y rebautizada Hospício Nacional de Alienados con la proclamación de la República, a partir de 1890 (Brasil, 11 jan. 1890). En aras de la uniformización, a lo largo de la nota, la denominaremos HNA.

${ }^{2}$ El Instituto de Psiquiatría surge del Pabellón de Observación, creado por el decreto n.1.559, de 7 de octubre de 1893 (Brasil, 7 out. 1893). Se constituía como puerta de entrada de "enfermos gratuitos, sospechosos de alienación mental, enviados por las autoridades públicas". Toda la documentación clínica del antiguo Pabellón de Observación permaneció en el Instituto de Psiquiatría. El Instituto Municipal de Asistencia a la Salud Nise da Silveira es heredero institucional de la Colônia do Engenho de Dentro (1911) que se destinó al trabajo y tratamiento de parte de la población femenina del HNA. El Instituto Municipal de Assistência à Saúde Juliano Moreira, por su parte, también puede considerarse heredero del antiguo hospicio. En la década de 1920, fue creada la nueva Colonia de Psicópatas Hombres (rebautizada como Colônia Juliano Moreira a partir de 1935) (Venancio, Cassilia, 2010). El Centro de Estudios e Investigaciones de la Escuela de Gestión Penitenciaria es el actual heredero de la documentación histórica del Manicomio Judiciario del Rio de Janeiro (creado en 1921). El manicomio acogió a los pacientes de la antigua "Sección Lombroso" del HNA (servicio de "alienados delincuentes" del Distrito Federal). Hoy, ese fondo guarda todos los documentos clínicos de los "alienados delincuentes" de la "Sección Lombroso" del HNA.

${ }^{3}$ Está en andamiento un convenio Fiocruz/Seap-RJ.

${ }^{4}$ Documentación para consulta en la Base de Dados História e Loucura: <http://historiaeloucura.gov.br/>.

\section{REFERENCIAS}

ABLARD, Jonathan D.

Madness in Buenos Aires: patients, psychiatrists and the Argentine State (1880-1983). Canada: University of Calgary Press. 2008.

BERRIOS, German E.

Melancolia e depressão durante o século XIX: uma história conceitual. Revista Latinoamericana de Psicopatologia Fundamental, v.15, n.3, p.590608. 2012.

\section{BRASIL.}

Decreto-lei n.7.055, de 18 de novembro de 1944. Cria o Centro Psiquiátrico Nacional e extingue o Conselho de Proteção aos Psicopatas e a Comissão Inspetora, no Ministério da Educação e Saúde, e dá outras providências. Disponível em: <https://www2.camara.leg.br/legin/fed/ declei/1940-1949/decreto-lei-7055-18-novembro1944-453631-publicacaooriginal-1-pe.html>. Acesso em: 14 fev. 2017. 18 nov. 1944.

BRASIL.

Decreto n.1559, de 7 de outubro de 1893. Reorganiza o serviço da Assistência Médico-legal de Alienados. Disponível em: <https://www2. camara.leg.br/legin/fed/decret/1824-1899/ decreto-1559-7-outubro-1893-502694publicacaooriginal-1-pe.html>. Acesso em: 12 fev. 2017. 7 out. 1893.

BRASIL.

Decreto n.142-A, de 11 de janeiro de 1890.

Desanexa do hospital da Santa Casa da Misericórdia desta Capital o Hospício de Pedro II, que passa a denominar-se Hospital Nacional de Alienados. Disponível em: <https://www2. camara.leg.br/legin/fed/decret/1824-1899/ decreto-142-a-11-janeiro-1890-513198publicacaooriginal-1-pe.html>. Acesso em: 12 fev. 2017. 11 jan. 1890.

BRASIL.

Decreto n.82, de 18 de julho de 1841 . Hospício de Pedro II anexado à administração do Hospital da Santa Casa da Misericórdia da Corte. Disponível em: <http://legis.senado.leg.br/ norma/385725/publicacao/15742236>. Acesso em: 15 fev. 2017. 18 jul. 1841.

COSTA, Jurandir Freire da.

História da psiquiatria no Brasil: um corte ideológico. Rio de Janeiro: Garamond. 2007.

CUNHA, Maria Clementina.

O espelho do mundo: Juquery, a história de um asilo. São Paulo: Paz e Terra. 1986.

DIAS, Allister.

Entre el pabellón y el hospicio: problemas y conflictos en la asistencia psiquiátrica en Rio de Janeiro de principios del siglo XX. Frenia, v.11, n.1, p.109-129. 2011.

DÖRRIES, Andrea; BEDDIES, Thomas.

The Wittenauer Heilstätten in Berlin: a case record study of psychiatric patients. In: Porter, Roy; Wright, David (Ed.). The confinement of the insane: international perspectives, 1800-1965. Cambridge: Cambridge University Press. p.149172. 2003. 
ENGEL, Magali.

Sexualidades interditadas: loucura e gênero masculino. História, Ciências, Saúde -

Manguinhos, v.15, supl., p.173-190. 2008.

ENGEL, Magali.

Os delírios da razão: médicos, loucos e hospício no Rio de Janeiro. Rio de Janeiro: Editora Fiocruz. 2001.

GONÇALVES, Monique.

Os primórdios da psiquiatria no Brasil: o Hospício Pedro II, as casas de saúde particulares e seus pressupostos epistemológicos (1850-1880). Revista Brasileira de História da Ciência, v.6, n.1, p.60-77. 2013.

HUERTAS, Rafael.

Historia cultural de la psiquiatría: (re)pensar la locura. Madrid: Catarata. 2012.

HUERTAS, Rafael.

Medicina social, control social y políticas del cuerpo: la subjetivación de la norma. In: Miranda, Maria; Sierra, Alvaro (Org.). Cuerpo, biopolítica y control social: América Latina y Europa en los siglos XIX y XX. Buenos Aires: Siglo XX. 2009.

LATOUR, Bruno.

La science en action: introduction à la sociologie des sciences. Paris: Gallimard. 1995.

MACHADO, Roberto et al.

Danação da norma: medicina social e a constituição da psiquiatria no Brasil. Rio de Janeiro: Graal. 1978.

MOLINA, Andrés.

La locura durante la Revolución Mexicana: los primeros años del manicomio general La Castañeda, 1910-1920. Ciudad de México: Colegio de México. 2009.

PEREIRA, Pamela Sant'Anna et al.

Listagem dos livros do Hospício Nacional de Alienados e instituições afins localizados no acervo do IMASJM. Rio de Janeiro: Instituto Municipal de Assistência à Saúde Juliano Moreira/Secretaria Municipal de Saúde-RJ;
Casa de Oswaldo Cruz/Fiocruz. Disponível em: <http://historiaeloucura.gov.br/>. Acesso em: 23 jul. 2019. 2016.

PORTER, Roy; WRIGHT, David (Ed.).

The confinement of the insane: international perspectives, 1800-1965. Cambridge: Cambridge University Press. 2003.

RIBEIRO, Daniele.

Ciência, cidade e redes de sociabilidade: o

Hospício de Pedro II em outras perspectivas. História, Ciência, Saúde - Manguinhos, v.23, n.4, p.1153-1167. 2016.

SACRISTÁN, Cristina.

Una valoración sobre el fracaso del manicomio de La Castañeda como institución terapéutica, 1910-1944. Secuencia: Revista de Historia y Ciencias Sociales, n.51, p.91-120. sept.-dic. 2001.

SCHORSKE, Carl.

Pensando com a história: indagações na passagem para o modernismo. São Paulo: Companhia das Letras. 2000.

SCULL, Andrew.

The insanity of place/the place of insanity: essays on the history of psychiatry. New York: Routledge. 2006.

TOMES, Nancy.

Feminist histories of psychiatry. In: Porter, Roy; Micaele, Mark S. Discovering the history of psychiatry. New York: Oxford University Press. p.348-383. 1994.

VENANCIO, Ana Teresa; CASSILIA, Janis. Política assistencial psiquiátrica e o caso da Colônia Juliano Moreira: exclusão e vida social (1940-1954). In: Wadi, Yonissa; Santos, Nádia (Org.). História e loucura: saberes, práticas e narrativas. Uberlândia: Edufu. 2010.

ZULAWSKI, Anne.

Doença mental e democracia na Bolívia: o Manicômio Pacheco, 1935-1950. In: Hochman, Gilberto; Armus, Diego. Cuidar, controlar, curar: ensaios históricos sobre saúde e doença na América Latina e Caribe. Rio de Janeiro: Editora Fiocruz. p.455-491. 2004.

\section{$\rightarrow \rightarrow+<<<$}

